\title{
Enhanced Behavioral Conditioning to Context and Impaired Behavioral and Neuroendocrine Responses to Conditioned Stimuli Following Ceruleocortical Noradrenergic Lesions: Support for an Attentional Hypothesis of Central Noradrenergic Function
}

\author{
Nathan R. W. Selden, ${ }^{1}$ Trevor W. Robbins, ${ }^{1}$ and Barry J. Everitt ${ }^{2}$ \\ Departments of ${ }^{1}$ Experimental Psychology and ${ }^{2}$ Anatomy, University of Cambridge, Cambridge, CB2 3EB, \\ United Kingdom
}

This study tested the hypothesis that cortical noradrenaline (NA) depletion induced by 6-hydroxydopamine (6-OHDA) widens attentional span, impairing the acquisition of conditioning to an explicit stimulus while enhancing conditioning to contextual stimuli. Sham-operated and NA-depleted rats were exposed to pairings of an auditory (clicker) CS and (footshock) US in a distinctive environment. Half of the lesioned and half of the control animals were trained with a short trace interval between presentations of clicker and shock, and half with a long trace. Associative learning theory predicts that a long trace interval should bias intact animals towards stronger contextual conditioning and, in contrast, a short trace interval should bias controls towards stronger CS conditioning.

During testing, NA-depleted animals showed impaired fear conditioning to explicit cues, compared with controls, indicated by their reduced suppression of drinking when the CS was introduced into a separate, lick-operant chamber. In contrast, the same animals exhibited enhanced fear of contextual cues, as shown by their greater preference for a "safe" environment over the one in which they were shocked. The behavioral and plasma corticosterone responses of individual animals to the CS were positively correlated in both the lesion and sham groups. Corticosterone levels corroborated the impairment in CS conditioning caused by the lesion. In contrast, behavioral and corticosterone responses to contextual stimuli were not significantly correlated in either group, and there was no enhancement of the corticosterone response to contextual stimuli in the lesioned animals. These findings indicate that the complex and seemingly opposing effects that can result from ceruleocortical NA depletion nevertheless support the proposal that noradrenergic projections are involved in the control of selective attention.

Received Feb. 27, 1989; revised June 16, 1989; accepted July 25, 1989

This work was supported by Project and Programme Grants from the Medical Research Council (U.K.) and a Wellcome Trust Major Award (to T.W.R. and B.J.E.). N.R.W.S. is a Marshall Scholar. We thank Prof. N. J. Mackintosh and Dr. A. Dickinson for discussion, and N. Martenz and A. Peard for technical assistance with the neuroendocrine assay.

Correspondence should be addressed to Dr. T. W. Robbins, Department of Experimental Psychology, University of Cambridge, Downing Street, Cambridge CB2 3EB, United Kingdom.

Copyright (C) 1990 Society for Neuroscience 0270-6474/90/100531-09\$02.00/0
Early proposals (Foote et al., 1975; Segal and Bloom, 1976) that activity in noradrenergic neurons, which reside in the locus ceruleus and project to their terminal regions via the dorsal noradrenergic bundle (DNAB), enhances the "signal-to-noise" ratio of evoked responses by forebrain neurons to environmental stimuli have becn bolstered by much recent elcetrophysiological evidence (Foote et al., 1980, 1983; Waterhouse and Woodward, 1980; Kasmatsu and Heggelund, 1982; AstonJones, 1985; Jacobs, 1987). However, these important neurophysiological studies do not, in themselves, argue for a particular functional role of noradrenergic transmission at a behavioral level. Thus, the physiological evidence is consistent with a role for central noradrenaline (NA) in consolidation and learning (Crow, 1968; Kety, 1970), anxiety (Redmond, 1979; Gray, 1982), or selective attention (Mason and Iversen, 1979; Mason and Lin, 1980; but see Pisa and Fibiger, 1983a, b), and each of these possibilities has been advanced in the behavioral literature.

The hypothesis that memory consolidation depends on telencephalic NA transmission is weakened by the well-documented lack of effect of central NA depletion on a wide variety of learning tasks (Mason and Iversen, 1975; Roberts, 1981; Robbins and Everitt, 1982; Robbins et al., 1985). The observation that NA depletion primarily affects the acquisition, but not the performance, of aversively motivated learning (Cole and Robbins, 1987) also compromises any simple role for NA in anxiety. Analysis of the role of ceruleocortical NA in selective attention has been more equivocal. Many studies of lesions to the DNAB have relied on indirect evidence, such as the presentation of distracting stimuli during operant tasks, and have obtained sometimes conflicting results (Roberts et al., 1976; Crow, 1978; Carli et al., 1983; Mason and Fibiger, 1978). Others have used more complex attentional paradigms, such as latent inhibition and blocking, to test the effects of DNAB lesions, but the results of these studies have also been equivocal and often contradictory (for a review, see Robbins et al., 1985; Everitt et al., 1989).

Recent work by animal learning theorists has emphasized the competitive nature of selective attention among salient cues. Thus, attention is the mechanism by which certain stimuli gain greater access to an associative mechanism to become more strongly associated with a reinforcer (Rescorla and Wagner, 1972; Mackintosh, 1975). This observation applies not only to competition between discrete, experimentally controlled stimuli such as lights and tones, but also to the competition between a single discrete stimulus and all of the contextual stimuli, such as the 
size and shape of an operant chamber or the noise of a ventilation fan, which are necessarily present in any learning situation (Odling-Smee, 1975a, b). In the present experiment, we adapted a procedure (Marlin, 1980) for examining the relative strength of conditioning to explicit cues (or CSs) and contextual cues in animals with 6-hydroxydopamine (6-OHDA)-induced lesions of the ceruleocortical noradrenergic projection and sham-operated controls.

Subjects were preexposed to a black/white, 2-chambered placepreference apparatus and then received CS-shock pairings while confined to the black chamber. By varying the temporal contiguity of CS and shock during this training (through the imposition of trace intervals of different durations between these stimuli), it is possible to demonstrate a reciprocal relationship between the strength of CS conditioning and the strength of contextual conditioning, as predicted by associative learning theory (Marlin, 1980). Thus, with a short trace interval, or gap, between the presentation of the CS and shock during training, subjects should exhibit strong CS and weak contextual conditioning during subsequent testing. With a long trace interval between $C S$ and shock during training, the opposite pattern of results should occur. Therefore, one group of lesioned animals and one group of sham-operated controls were trained using a long $(30 \mathrm{sec})$ trace interval and one group of each were trained with a short $(10 \mathrm{sec})$ trace interval. Subsequent tests allowed for the measurement of fear conditioning in the presence of the CS alone and in the presence of contextual cues alone. The test for CS conditioning involved the suppression of licking during CS presentation in a separate lick-operant chamber. Contextual conditioning was measured in the same animals in a test of relative preference for the white or black compartment of the original training apparatus.

In addition to these behavioral indices of conditioning, we also used a neuroendocrine measure of fear conditioning, the concentration of plasma corticosterone. Corticosterone secretion is well known to increase in response to a variety of physical, as well as psychological, stressors. Furthermore, noradrenergic projections from both the locus ceruleus and other NA cell groups in the medulla to the hypothalamus have been implicated in the regulation of corticotrophin-releasing factor (CRF), and hence ACTH and corticosterone secretion both basally and under conditions of stress (Fuxe et al., 1973; Meites and Sonntag, 1981; Gillies and Lowry, 1986). Although the majority of the hypothalamic NA innervation projects via the ventral noradrenergic bundle component of the central tegmental tract, rather than the DNAB, the effects of lesions to the latter were also assessed in terms of the relationship between behavioral and plasma corticosterone responses to an aversive CS and context.

\section{Materials and Methods}

\section{Subjects}

Subjects were 34 Lister hooded male rats (OLAC, Bicester, U.K.) weighing between 220 and $250 \mathrm{gm}$ at the beginning of the experiment. They were housed, 2 per cage, in a temperature-controlled room $\left(22^{\circ} \mathrm{C}\right)$ under natural daylight. All subjects were handled in squads of 4 for 4 min each day, for $3 \mathrm{~d}$ before and $3 \mathrm{~d}$ after surgery. Food and water were freely available in the period before and during recovery from surgery.

\section{Apparatus}

Two lick-operant chambers and one place-preference apparatus were used. The operant chambers $(25 \times 22 \times 21 \mathrm{~cm})$ were constructed of 3 brown, opaque Plexiglas walls, a white Plexiglas ceiling, and a fourth, clear Plexiglas wall, which also served as a door. The floor was a shock grid with bars separated by $1 \mathrm{~cm}, 4 \mathrm{~cm}$ above a sawdust pan. In the center of the ceiling was a single, $2.8 \mathrm{~W}$ house light, which remained lit throughout the session. In the center of one wall adjacent to the door, and $8 \mathrm{~cm}$ above the grid floor, a drinking spout cquipped with a lickometer circuit protruded $1.5 \mathrm{~cm}$ into the chamber. All but the last 2 $\mathrm{mm}$ of the spout was covered in thick plastic so that only licks at the aperture itself would be recorded by the lickometer. On the outside of the opposite wall was a heavy-duty relay, used to present the "clicker" CS. Each chamber was enclosed in a large $(50 \times 48 \times 32 \mathrm{~cm})$, wooden sound-attenuating box, which provided masking noise from an extractor fan.

The place-preference apparatus consisted of $2(13 \times 40 \times 32 \mathrm{~cm})$ chambers separated by a small central chamber $(13 \times 14 \times 30 \mathrm{~cm})$. Photocell beams at the borders between the small central chamber and the 2 main chambers enabled on-line monitoring of a subject's location. The walls of one chamber and the adjacent half of the small central chamber were covered in white cardboard. The remaining walls were covcred in black cardboard. The floor was a shock grid with bars $1 \mathrm{~cm}$ apart, $3 \mathrm{~cm}$ above a silver sawdust tray. The tray was always full in the black compartment and empty in the white. Illumination was provided by a single, $2.8 \mathrm{~W}$ lamp in the center of the long wall of each of the two main chambers, which remained illuminated throughout the session. A heavy cardboard door (white and black on the appropriate sides) split the small central chamber and extended from the top of the box to 8 $\mathrm{cm}$ above the grid floor when open and to the bottom of the sawdust tray when closed. On the outside of the long wall of the black chamber was a heavy-duty relay, identical to those attached to the lick-operant chambers, which provided a "clicker" that served as the CS. Ceilings were provided by thick wooden planks, covered in the appropriate shading of cardboard.

All apparatus was controlled on-line by an Acorn System 3 microcomputer. The CS was provided by switching the heavy-duty relays on and off at a frequency of $4 \mathrm{~Hz}$. The reinforcer was shock, delivered through the grid floor of the place-preference apparatus by a Grason Stadler scrambled-footshock generator (model E6070B).

\section{Surgical procedure}

Rats in experimental and control groups were pretreated $30 \mathrm{~min}$ before surgery with an i.p. injection of $50 \mathrm{mg} / \mathrm{kg}$ pargyline hydrochloride (Sigma Chemical Co.) (see Breese and Taylor, 1971). Rats received bilateral injections of either the neurotoxin 6-OHDA hydrobromide (Sigma Chemical Co.) or the vehicle $(0.1 \%$ ascorbic acid dissolved in $0.9 \%$ isotonic saline) under Avertin anesthesia $(10 \mathrm{ml} / \mathrm{kg})$. Solutions of neurotoxin were prepared in ice-cold vehicle just before injection and kept at $4^{\circ} \mathrm{C}$ in the dark. Injections were made using a $10-\mu 1$ Hamilton syringe, mounted on a Harvard infusion pump, and connected by PE10 polythene tubing to a 30-gauge stainless steel cannula. The coordinates for injection were (in $\mathrm{mm}$ ): bregma -6.0 , lateral +1.0 , ventral -5.0 from dura (with the incisor bar $2.4 \mathrm{~mm}$ below the interaural line). Rats were injected bilaterally over 4 min with a $2 \mu \mathrm{l} /$ side of 6 -OHDA solution at a concentration of $2 \mu \mathrm{g} / \mu \mathrm{l}$ (free base) or the vehicle alone. Cannulae were left in place for 2 min after infusions were completed. Animals were allowed 3 weeks to recover from the surgery before the beginning of behavioral lesting.

\section{Biochemical procedure}

At the end of the experiment, animals were stunned and decapitated, their brains rapidly removed and dissected on ice. The following areas were taken: (1) a slice of cortex running from frontal to occipital poles; (2) dorsal and ventral hippocampus (together); (3) hypothalamus (coronal cuts behind the optic chiasm and in front of the mammillary bodies, lateral cuts immediately lateral to the descending fornix, and a horizontal cut along the top of the third ventricle); (4) septum (medial and lateral); and (5) amygdala.

NA concentrations were measured in all areas using high-performance liquid chromatography (HPLC) with electrochemical detection (ECD), following the method of Mefford (1981). However, in hypothalamic tissue, NA, dopamine, and adrenaline were assayed simultaneously by HPLC with ECD, following the same method.

Blood samples were kept on ice and then centrifuged at $3000 \mathrm{rpm}$ for $10 \mathrm{~min}$. Serum was stored at $-20^{\circ} \mathrm{C}$ until assayed. At that time, samples ( $1 \mu \mathrm{l}$ serum) in duplicate were extracted with dichloromethane. Solvent extract was evaporated under a vacuum at $25^{\circ} \mathrm{C}$ and the samples redissolved in buffer $(0.05 \mathrm{M}$ phosphate, $\mathrm{pH} 7.4$, containing $0.9 \% \mathrm{NaCl}$, 
Table 1. Means \pm SEM of NA concentrations in the brains of DNAB-lesioned and sham-operated rats

\begin{tabular}{llll} 
Tissue & \multicolumn{2}{l}{ Concentrations } & $\begin{array}{l}\text { Mean } \\
\text { depletion } \\
(\%)\end{array}$ \\
\cline { 2 - 4 } & DNAB & Sham & 100 \\
Neocortex & $0.000 \pm 0.000$ & $0.282 \pm 0.010$ & 100 \\
Hippocampus & $0.000 \pm 0.000$ & $0.242 \pm 0.011$ & 96 \\
Amygdala & $0.015 \pm 0.006$ & $0.380 \pm 0.010$ & 79 \\
Septum & $0.174 \pm 0.030$ & $0.818 \pm 0.040$ & 72 \\
Hypothalamus & $0.979 \pm 0.056$ & $3.473 \pm 0.171$ &
\end{tabular}

Values are expressed as $\mathrm{ng} / \mathrm{mg}$ of wet tissue weight. The lesion resulted in highly significant depletions $(p<0.001)$ in all structures assayed.

$1 \%$ gelatin, and $0.0001 \%$ phenol red). Corticosterone was measured in the extracts by ELISA using an antiserum raised against corticosterone3-carboxy-methyloxime conjugated to thyroglobulin (Bioclin, U.K.) and a corticosterone-3-carboxymethyloxime-HRP conjugate as label, using $O$-phenylenediamine as substrate. Sensitivity of the assay was $27 \mathrm{ng} /$ $\mathrm{ml}$ and recovery (assessed by the inclusion of trace amounts of ${ }^{3} \mathrm{H}$ corticosterone) was $89 \%$. The intra-assay coefficient of variation averaged $4.3 \%$.

\section{Behavioral procedure}

Rats were water deprived $72 \mathrm{hr}$ prior to the beginning of the behavioral experiment and given $30 \mathrm{~min}$ access to water each evening between 6 and 8 p.m. On the third day of the deprivation schedule, the amount of water drunk by each pair of NA-depleted or sham-operated rats in the home cage in 30 min was measured.

Day $I$. In the morning, animals were placed individually into the lick-operant chambers. They were removed from the chamber $1 \mathrm{~min}$ after making their first lick and returned to the home cage. The latency to lick and number of licks made were recorded. This procedure was repeated for each animal in the afternoon.

Day 2. Animals were placed individually into the white chamber of the place-preference apparatus, facing the central passage, which was open, and were allowed to explore freely for $5 \mathrm{~min}$. Baseline blackversus-white preference was recorded as the number of seconds spent in the white chamber. Each rat was removed after $5 \mathrm{~min}$, the door between chambers closed, and the rat immediately replaced in the black chamber, where it received 5 pairings of a $30 \mathrm{sec}$ clicker CS and a 0.5 sec, $0.5 \mathrm{~mA}$, scrambled footshock. One pairing was presented at a random time within each of five, $4 \mathrm{~min}$ bins in a $20 \mathrm{~min}$ session. One group of 9 lesioned animals and one group of 8 sham-operated controls received training with a $10 \mathrm{sec}$ trace interval between the offset of the CS and the onset of the shock, and separate groups were trained with a 30 sec trace interval. After conditioning, the passage between compartments was opened again, and the rats were allowed to explore for $5 \mathrm{~min}$ to prevent freezing during testing, as recommended by Odling-Smee (1975a).

Day 3. CS suppression testing was carried out in the lick-operant chamber. Each rat was placed in the chamber with the house light illuminated. The fifth lick emitted triggered the onset of the clicker CS, which remained on for $15 \mathrm{~min}$. The latency to the first lick, to the 5 th lick, and to the 40th lick, and the number of licks in the first min of CS were recorded. Immediately after testing, each rat was anesthetized with $10 \mathrm{ml} / \mathrm{kg}$ Avertin and a $1 \mathrm{ml}$ sample of blood was removed by cardiac puncture, using a 23 gauge, $25 \mathrm{~mm}$ needle, washed in advance with heparin (1000 units/ml; Sigma Chemical Co.). No blood samples were collected more than 3 min after completion of the test session.

Day 5. Contextual conditioning testing was carried out in the placepreference apparatus. Each rat was placed individually in the white chamber facing the central passage, which was open, and was allowed to move freely between chambers for $5 \mathrm{~min}$. The number of seconds spent on the white (nonshock) side was recorded. After testing, each rat was returned to the home cage for $10 \mathrm{~min}$ before blood samples were taken as described for day 3 above. Thus, blood samples were removed approximately $16 \mathrm{~min}$ after the first exposure to aversive stimuli on both days 3 and 5 .

Day 7. Animals were given a $20 \mathrm{~min}$ reconditioning session (as on day 2 , above) followed immediately by the collection of a blood sample (as on day 3, above).

Day 12. Animals were given a second suppression test in the lickoperant chamber. At the beginning of this test session, the houselight was off. The 5th lick emitted by each subject triggered the onset of a novel visual stimulus. This stimulus was provided by flashing the houselight at a frequency of $2.5 \mathrm{~Hz}$. The latency to the first lick to the 5 th lick and to the 40th lick was recorded.

At the time of death, blood samples were collected (only from the first animal in each cage pair, to control for possiblc order cffcets) immediately upon removal from the home cage.

All behavioral testing and blood sampling took place between 8 a.m. and 2 p.m. CS-shock training took place between 2 p.m. and 10 p.m. Animals were allowed to drink freely during each evening following blood sampling.

\section{Statistical analysis}

Behavioral and neuroendocrine measures, as described above, were subjected to analysis of variance (ANOVA) using GENSTAT (Rothamstead Experimental Station). When interactions were found, further analysis was made of the simple main effects. Certain measures, noted below, were also subjected to correlational analysis by least-squares method or to nonparametric analysis using the Information Statistic (Kullback, 1968). Neurochemical data were compared using Student's $t$ tests.

\section{Results}

Neurochemical

Neurochemical data for all lesioned animals and 9 randomly selected sham animals are shown in Table 1.6-OHDA injection significantly reduced NA levels in all 5 areas assayed. Cortical and hippocampal NA was reduced to undetectable levels, while small, detectable amounts of NA remained in the amygdalae of 7 lesioned animals. The level of hypothalamic and of septal NA in the lesioned animals was $28 \%$ and $21 \%$ of control values, respectively. There were no significant effects of the lesion on the levels of dopamine (means: lesion, $0.242 \pm 0.018 \mathrm{ng} / \mathrm{mg}$; sham, $0.260 \pm 0.037 \mathrm{ng} / \mathrm{mg}$; $\mathrm{ns}$ ) or adrenaline (means: lesion, $0.032 \pm 0.003 \mathrm{ng} / \mathrm{mg}$; sham, $0.038 \pm 0.009 \mathrm{ng} / \mathrm{mg}$; ns) in hypothalamus.

\section{Behavioral}

\section{Effects of DNAB lesions on the acquisition of fear conditioning} to the CS

CS conditioning data were evaluated using the latency to 40th lick (35th lick in the presence of the CS) as defined by Marlin (1980). The incidence and failure of conditioning to the CS are shown in Figure 1. Failure to condition was defined as making more than 35 licks during the first minute of CS presentation. In the $30 \mathrm{sec}$ trace condition, DNAB-lesioned animals were significantly less likely to condition to the CS than shams, $\chi^{2}(1)$ $=10.7, p<0.005$. The difference between lesioned and sham animals in the $10 \mathrm{sec}$ trace condition was in the same direction but did not reach significance, $\chi^{2}(1)=1.3$, ns. Analysis of the dependent variable used by Marlin (1980), the latency from the 5th lick (onset of CS) to the 40th lick, yielded the same pattern of results. Thus, there was a significant lesion by condition interaction, $F(1,30)=6.482, p<0.02$. This interaction was due to shorter latencies to the 40th lick in DNAB-lesioned animals than in shams in the $30 \mathrm{sec}$ trace condition [means: lesion, 71 $\pm 27 \mathrm{sec}$; sham, $262 \pm 54 \mathrm{sec} ; F(1,15)=11.344, p<0.005]$. There was no significant difference between the latency to the 40th lick in lesion and sham groups in the $10 \mathrm{sec}$ trace condition. [means: lesion, $182 \pm 65 \mathrm{sec}$; sham, $150 \pm 28 \mathrm{sec} ; F(1,15)=$ $0.446, \mathrm{~ns}]$. There were no simple main effects of trace interval condition in either the lesion or control groups. 

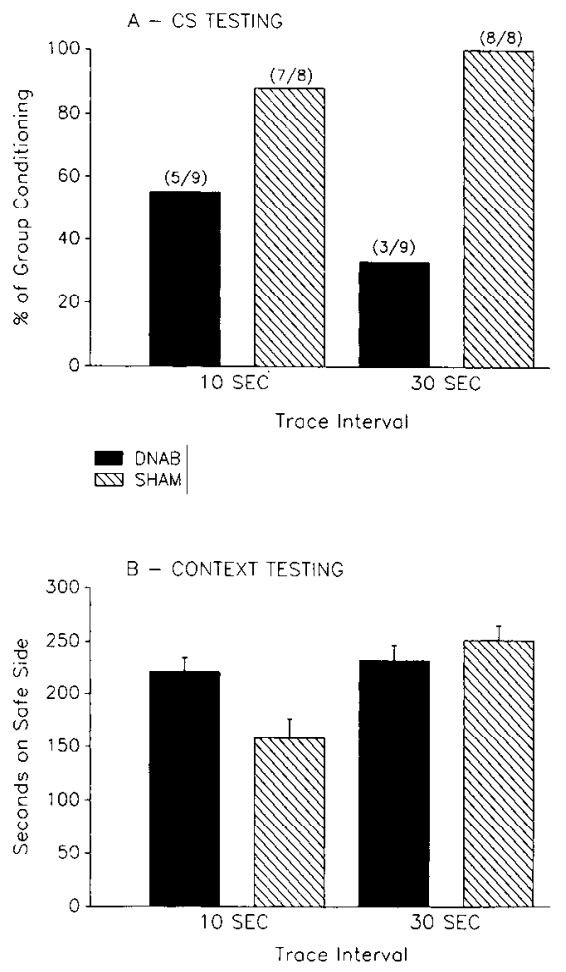

Figure 1. A, Proportion of DNAB-lesioned and sham-operated rats that made fewer than 35 licks during the first minute of CS presentation (see Results) is shown separately for the 10 and $30 \mathrm{sec}$ trace interval groups. The number of rats meeting this criterion, and the total number of rats in each group, is also indicated, in parentheses. $B$, The strength of context conditioning measured as the number of seconds spent on the nonshock side of the place-preference apparatus during a 300 second test interval. The data shown in $A$ and $B$ were gathered in separate tests carried out on the same animals. Error bars represent 1 SEM.

A number of control measures for nonassociative effects on performance were also analyzed. There was no difference between lesion and sham groups in the amount of water consumed during $30 \mathrm{~min}$ in the home cage, after $23.5 \mathrm{hr}$ water deprivation (means: lesion, $23.3 \pm 0.7 \mathrm{ml} /$ cage; sham, $24.3 \pm 1.2 \mathrm{ml} /$ cage; ns). There was no difference between lesion and sham groups, and no interaction with trace condition, for the latency to make the first lick during the second lick-operant chamber preexposure (means: lesion, $22.8 \pm 7.0 \mathrm{sec}$; sham, $16.2 \pm 4.5 \mathrm{sec}$; ns); for the total number of licks during that preexposure (means: lesion, $128 \pm 20 \mathrm{sec}$; sham, $133 \pm 18$; ns); or for the latency to the first, pre-CS lick in the operant chamber during the CS test session (means: lesion, $11.8 \pm 2.8 \mathrm{sec}$; sham, $13.5 \pm 4.4$ sec; ns). Finally, there was no difference between NA-depleted and control animals in the latency to lick in the presence of a novel, but motivationally irrelevant stimulus, i.e., one that has not been paired with shock (day 12) (means: lesion, $32.8 \pm 4.1$ sec; sham, $23.4 \pm 4.4 \mathrm{sec}$; ns). The grand mean latency to 40 th lick in this situation, for all groups, was $29 \mathrm{sec}$, i.e., considerably shorter than the latencies observed during CS testing on day 5 .

In summary, DNAB lesions significantly and selectively impaired the acquisition of aversive CS conditioning.

\section{Effects of DNAB lesions on the acquisition of fear conditioning to the context}

Figure 1 also shows the contextual conditioning exhibited by both groups during testing on day 5 , measured as time spent in

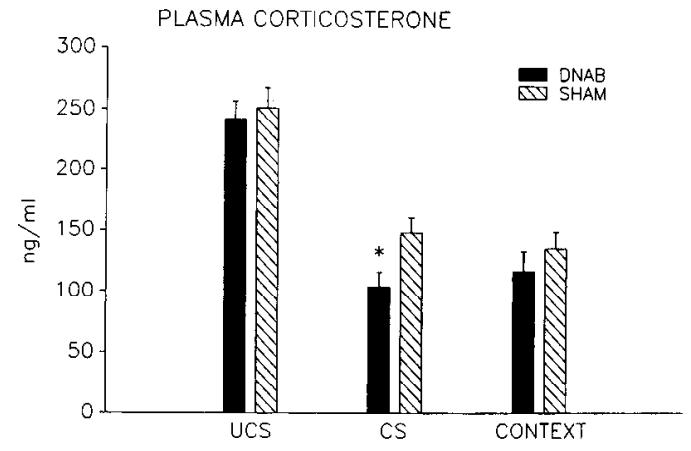

Figure 2. Mean plasma corticosterone levels in DNAB-lesioned and sham-operated rats after reexposure to CS-shock pairings on day 7 (UCS), after CS testing on day $3(C S)$, and after context testing on day $5(C O N T E X T)$. Because there was no effect on plasma corticosterone of trace interval condition, and no interaction between lesion and this factor (see Results), data were collapsed over the 10 and $30 \mathrm{sec}$ trace groups. The asterisk indicates difference from sham-operated control, $p<0.02$. Error bars represent 1 SEM.

the white (safe) chamber of the place-preference apparatus. Inspection of the individual data revealed that, despite employing Odling-Smee's (1975a) anti-freezing procedure, one lesioned animal froze upon first entry into the black compartment. This resulted in a preference score that was a statistically significant outlier using Grubbs' test (1969): $p<0.05$. The data for this animal were discarded, although inclusion would not have affected the pattern or significance of any results. Analysis of the time spent during testing in the safe compartment revealed a highly significant lesion by condition interaction, $F(1,30)=7.491$, $p=0.01$. Whereas in the $10 \mathrm{sec}$ trace condition lesioned animals spent more time than shams in the safe compartment $[F(1,15)$ $=8.068, p<0.02]$, there was no difference between lesion and sham groups in the $30 \mathrm{sec}$ trace condition $[\mathrm{F}(1,15)=0.954$, ns] There was no difference between lesion groups in the 10 and 30 sec trace conditions $[F(1,17)=0.318, \mathrm{~ns}]$, but controls in the $30 \mathrm{sec}$ condition spent more time in the safe compartment than controls in the $10 \mathrm{sec}$ condition $[F(1,13)=16.08, p=0.001]$.

The amount of time spent in the white compartment during preexposure on day 2 was also analyzed as a control measure. There were no differences between groups and no interactions [means: lesion, $140 \pm 9 \mathrm{sec}$; sham, $144 \pm 7 \mathrm{sec}$; ns].

In summary, DNAB lesions enhanced aversive contextual conditioning in the $10 \mathrm{sec}$, but not the $30 \mathrm{sec}$ trace condition, probably due to ceiling effects in the controls in the latter case.

\section{Effects of the lesion on the plasma corticosterone response to fear conditioning}

Plasma corticosterone levels from blood samples collected during the behavioral experiments are shown in Figure 2. A separate analysis was made for each group of samples (CS, UCS, and context), treating both lesion and trace condition as factors. However, since there were no significant lesion $\times$ condition interactions, there were no differential effects of the DNAB lesion on the 10 and $30 \mathrm{sec}$ trace conditions. Consequently, mean corticosterone levels for the DNAB and sham groups are collapsed over the two trace interval conditions in Figure 2. The lesion did not affect corticosterone levels measured after context testing on day $5[F(1,30)=0.733$, ns] or after CS-shock retraining on day $7[F(1,30)=0.230, n s]$. NA depletion did lower the corticosterone response of lesioned animals to CS testing on day 
$3[F(1,30)=7.233, p<0.02]$. Levels of corticosterone in home cage blood samples collected at the time of sacrifice did not differ (means: lesion, $44 \pm 7 \mathrm{ng} / \mathrm{mg}$; sham, $48 \pm 5 \mathrm{ng} / \mathrm{mg}$; ns).

Further analysis of the corticosterone data revealed a strong correlation, within both lesion and sham groups, between the strength of CS conditioning, as measured by latency to the 40th lick, and the corticosterone response to CS testing, in individual animals: lesion, $r(18)=0.62, p<0.01$; sham, $r(16)=0.76, p$ $<0.001$. There was no difference between the regression lines for the lesion and sham groups $(t=0.116, \mathrm{~ns})$. These correlational data are illustrated in Figure 3. In contrast, there was no significant correlation, in any group, between the strength of context conditioning, as measured by time spent in the safe compartment, and the corticosterone response to context testing $[r(34)=0.07$, ns; Fig. 3].

In summary, the plasma corticosterone response was unaltered in the DNAB group, except when it was reduced in the case of testing CS conditioning.

\section{Correlational analysis of the strength of fear conditioning to CS and contextual cues}

Figure 4 shows scatter plots of the relationship between strength of CS conditioning (seconds to 40th lick) and strength of context conditioning (lime in the safe compartment) for all $\mathbf{4}$ groups in the experiment. Only sham animals in the $30 \mathrm{sec}$ trace condition showed a significant correlation between these measures: $r(8)=$ $-0.86, p<0.01$. While this association may appear to depend on a single point near the upper limit of the abscissa, it is nevertheless still significant at the $5 \%$ level, even with this point excluded. Thus, at least in the sham group trained with a 30 sec trace interval, the degree of CS conditioning was inversely related to the degree of contextual conditioning.

\section{Discussion}

The results of this experiment have shown a dissociation between the effects of ceruleocortical NA depletion on fear conditioning to explicit cues, or CSs, and conditioning to diffuse, contextual cues. In the same experiment, DNAB-lesioned animals showed impaired acquisition of conditioned suppression to an auditory CS which had been previously associated with shock and enhanced acquisition of conditioned fear to the contextual cues in the chamber where shock occurred (Fig. 1).

\section{Corticosterone response to fear conditioning}

The reduced level of fear conditioning to the CS in lesioned animals was evident from both behavioral and neuroendocrine measures (Figs. 1, 2). The behavioral and corticosterone responses to conditioned stimuli were highly correlated and, to an equal extent, in both lesioned and control animals. Thus, despite the hypothalamic depletion of NA in the DNAB group, it appears that both deficits in the lesioned animals reflect an underlying impairment in fear conditioning and that there is no direct effect of the lesion on the hypothalamic-pituitary-adrenal axis. The observation that DNAB-lesioned animals exhibited a normal corticosterone response, at significantly higher absolute levels, when reexposed to CS-shock pairings on day 7, clcarly indicates that the noradrenergic lesions did not disrupt the neuroendocrine mechanisms regulating the adrenal cortex. This conclusion is consistent with the finding of Martin-Iverson et al. (1982) that DNAB lesions did not alter either basal or stressinduced corticosterone secretion.

One implication of these observations is that the pattern of
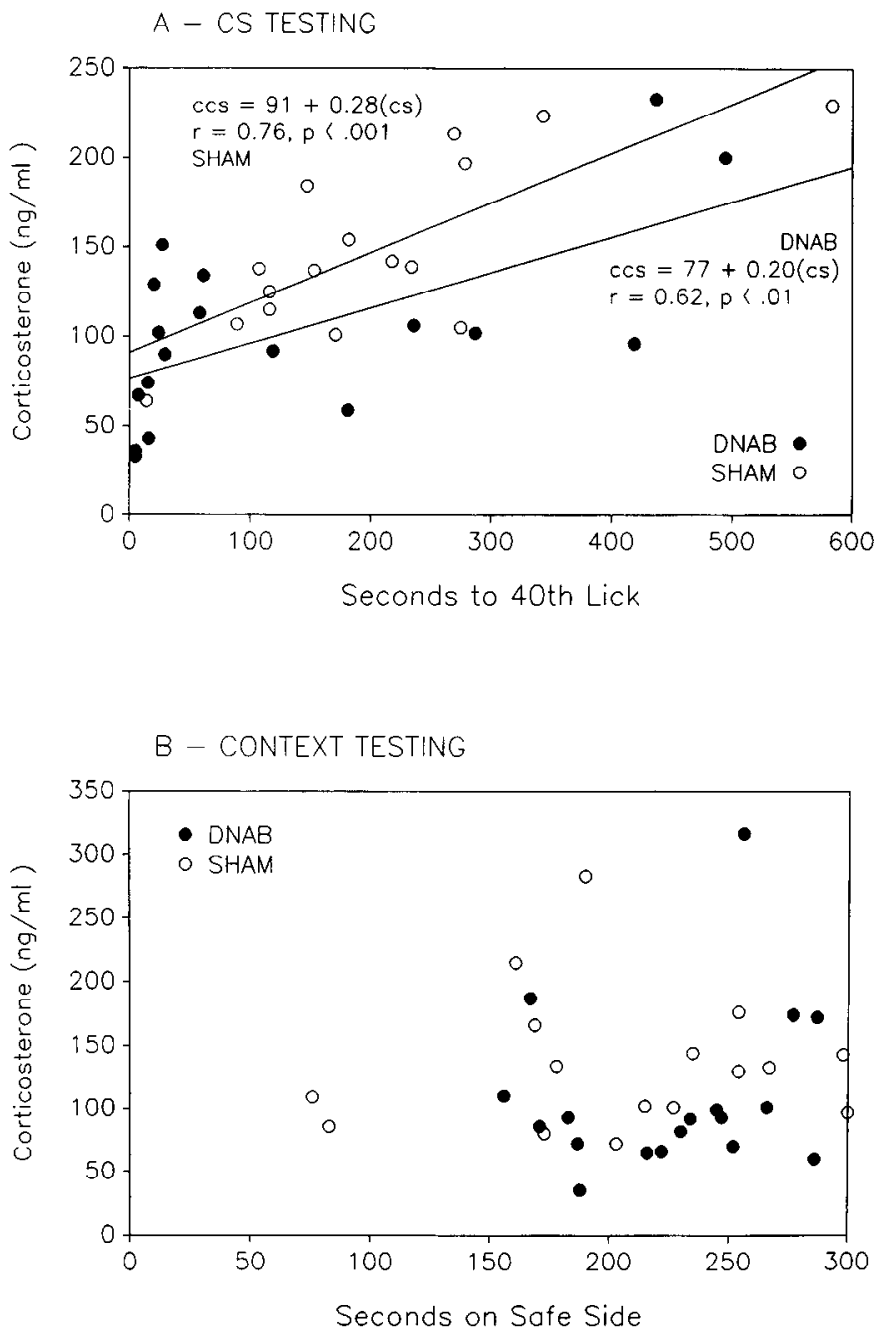

Figure 3. Relationship between behavioral ( $x$-axis) and plasma corticosterone ( $y$-axis) responses to CS testing $(A)$ and context testing $(B)$ for DNAB-lesioned and sham-operated rats. Best-fit lines for the DNAB and sham groups in $A$ are plotted separately; these lines do not differ significantly from each other. CCS, corticosterone; $r$, Pearson's rho.

hypothalamic NA loss following DNAB lesions does not have any impact on CRF neurosecretory mechanisms within the paraventricular nucleus (PVN) and median eminence (see Gillies and Lowry, 1986). Although the noradrenergic innervation of the PVN arises principally from medullary A1 and A2 cell groups via projections running in the central tegmental tract, a substantial proportion of these axons runs to the hypothalamus via the DNAB (Sawchenko and Swanson, 1982). Variable damage to these, as well as ceruleohypothalamic axons in the DNAB, can have neuroendocrine consequences (e.g., the vasopressin response to hemorrhage is markedly diminished, see Lightman et al., 1983). However, it appears that any noradrenergic modulation of hypothalamus-pituitary-adrenal responses to the stressors used in these experiments does not involve axons which travel via the DNAB at the midbrain level where 6-OHDA injections used to lesion this projection were sited. Nevertheless, the fact that responses of hippocampal neurons to their noradrenergic input may be affected by corticosterone (Roberts, 1981) suggests that direct noradrenergic influences on the release of $\mathrm{CRF}$, and thus ACTH and corticosterone, should be investigated carefully in the context of aversive learning. The rich adrenergic 


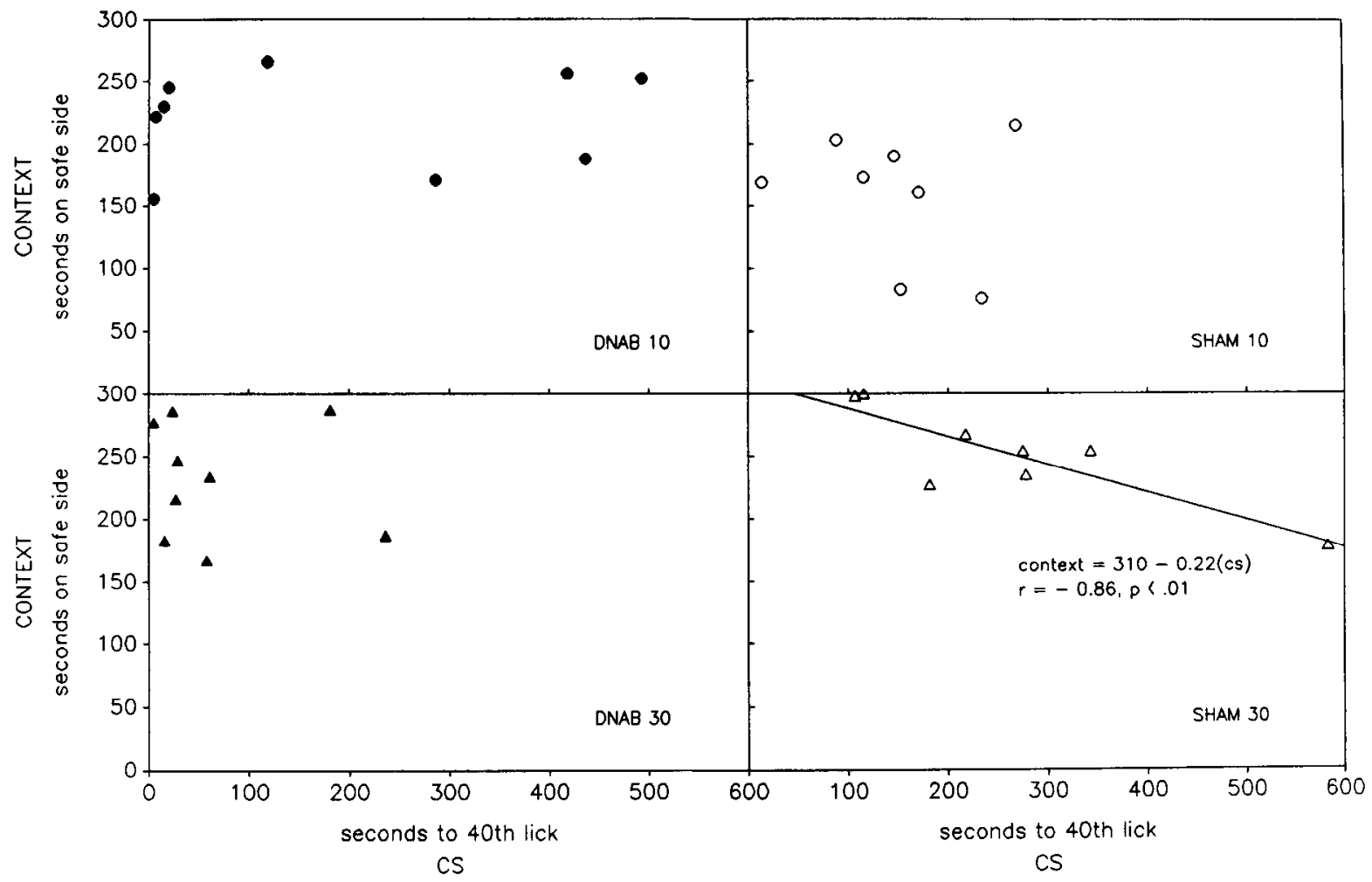

Figure 4. Relationship between CS conditioning ( $x$-axis) and context conditioning ( $y$-axis) for DNAB-lesioned and sham-operated rats, in the 10 and $30 \mathrm{sec}$ trace interval groups. The best-fit line shown from sham-operated rats in the $30 \mathrm{sec}$ trace interval condition accounts for approximately $74 \%$ of the variance, as indicated by Pearson's correlation coefficient, $r$.

innervation of the parvicellular paraventricular nucleus (Hökfelt et al., 1984; Sawchenko and Swanson, 1982) may also prove to be important in stress-induced CRF release (Mezey et al., 1984).

\section{Ceruleocortical noradrenaline and attention}

The present results add to a substantial body of evidence showing deficits in NA-depleted animals in the acquisition of aversive conditioned suppression (Tsaltas et al., 1983, 1984, 1989; Cole and Robbins, 1987). The previous literature has not always shown consistent effects of DNAB lesions on aversive conditioning (e.g., Lorden et al., 1980), but this is probably due to differences between experiments in the behavioral procedures used (see Cole and Robbins, 1987; N. R. W. Selden, B. J. Everitt, and T. W. Robbins, unpublished observations) and, possibly, in the extent of NA depletion in telencephalic sites. The opposite effects of the DNAB lesions on $\mathrm{CS}$ and contextual aversive conditioning shown here, in the same experiment, clearly indicate the importance of variations in behavioral testing procedure.

In theoretical terms, the dissociation between the effects of NA depletion on conditioning to explicit and contextual cues contradicts theories which ascribe to central NA functions of consolidation or reinforcement in learning (Kety, 1970; Crow, 1968). Thus, the latter theories predict deficits in both types of conditioning. Similarly, Gray's (1982) theory of anxiety and septohippocampal function, which argues that ceruleal projections to the hippocampus are important for the encoding of signals of punishment and nonreward, and Redmond's (1979) theory of the effects of fearful stimuli mediated by NA projections from the LC predict some degree of impairment in the acquisition and performance of any aversively motivated learning task, regardless of the nature of the conditioned cues. The present results, then, clearly constrain both these classes of theory.

On the other hand, the results of this study are entirely consistent with a theory that posits a role for the rostral noradrenergic projections from the LC in the maintenance of selective attention. Similar hypotheses, advanced in the past, have generated a good deal of indirect evidence for such a proposal (Mason and Iversen, 1979; Mason and Lin, 1980; but see Robbins and Everitt, 1982; Pisa and Fibiger, 1983a, b).

The impaired CS conditioning and enhanced contextual conditioning seen in the DNAB groups in the present cxpcriment can be explained most parsimoniously as a broadening of cue utilization (or attention) during conditioning, which becomes evident during the test phase. Thus, the effect of the DNAB lesion hypothetically occurs during the conditioning phase. It is possible, of course, that there are effects during other stages of the experiment, perhaps during preexposure to the place-preference apparatus or during testing. For example, DNAB lesions have been proposed to retard habituation to alarming or potentially aversive stimuli, such as the bright alley of a maze (Pisa and Fibiger, 1983b), and this might have affected behavior during place-preference testing. In addition, DNAB lesions may have reduced conditioned suppression shown to a CS as a consequence of reduced behavioral inhibition (Gray, 1982) to cues which have been normally conditioned to the UCS. However, neither of these alternative accounts of the data is supported by other observations. First, it is unclear what prediction would be made concerning preexposure and habituation to the place- 
preference apparatus. Retarded habituation to the white side could exaggerate preference for the black side and thus apparently reduce contextual conditioning, which would make it more difficult to demonstrate the enhanced contextual conditioning seen here. On the other hand, the effect of retarded habituation might eventually be to increase exploration of the previously aversive white side, and thus apparently enhance contextual conditioning. Nor can this latter possibility easily account for our results. First, the white side preference of lesioned and sham rats in the present experiment, during preexposure, was virtually identical. Second, a separate control experiment (Selden et al., unpublished observations) has shown no effect of DNAB lesions on habituation to the white side, during either initial or repeated exposures to this place-preference apparatus.

An explanation of the effects of impaired CS conditioning as a consequence of behavioral disinhibition in the test (and not the acquisition) phase of the experiment is also compromised by evidence that DNAB lesions only affect aversive conditioning if the lesion is made prior to and not following training (Cole and Robbins, 1987). Thus, it is most likely that the present effects of DNAB lesions occur during the conditioning phase.

Recently, Tsaltas et al. (1989) have attempted to test the effects of DNAB lesions on aversive conditioning to contextual stimuli. They measured the suppression of food-reinforced lever pressing during the presentation of an "envelope" stimulus (flashing light), which contained presentations of a "punctate" CS (auditory clicker) that served as either a strong or weak predictor of the occurrence of foot shock. Tsaltas et al. (1989) found no effect of NA depletion on conditioning to the envelope stimulus with either strongly or wcakly predictive CSs. However, the authors themselves note that their operational definition of context, as a single, temporally discrete and modality-specific stimulus, "differs radically" from the definition of contextual cues used in the human literature (Tsaltas et al., 1989, p. 253) and also from the operational and conceptual definition of context in the present experiment (see also Nadel and Willner, 1980, for a discussion of these issues).

As described above, the present experiment was based on a general model of stimulus competition, or attention, in associative learning (Kamin, 1969; Rescorla and Wagner, 1972; Mackintosh, 1975). The reciprocal relationship between the strength of conditioning to CS and to context in individual animals reported here (Fig. 4) lends support to this model. Thus, an individual animal that conditions strongly to the CS conditions weakly to context, and vice versa. This is probably the result of competition between cues for access to an associative mechanism during learning. Attentional processes should optimally select the most relevant and predictive stimuli in the environment for association with a given reinforcer. This relationship between the strength of conditioning to explicit and contextual cues in individual animals, while following directly from the predictions of animal learning theorists (Kamin, 1969; Rescorla and Wagner, 1972), has, to our knowledge, never before been confirmed experimentally.

The predicted negative correlation between conditioning to explicit and contextual cues was not observed in either of the $10 \mathrm{sec}$ trace groups, indicating that the effect can only be demonstrated within certain parameters. Further, the effect is not seen in the DNAB-lesioned animals in the $30 \mathrm{sec}$ trace condition. This is probably not the result of some fundamental associative impairment but, rather, of a floor effect in CS conditioning in the lesioned rats, which does not allow sufficient variances to demonstrate a correlation.

\section{Dual processes and neural systems}

There are, however, alternative interpretations of the contrasting effects of DNAB lesions on CS and contextual conditioning, the most compelling of which is that they reflect changes in not 1 , but 2, distinct processes. First, it can be argued that these effects do not actually reflect a broadening of attention because the differences between lesioned and control animals in CS and contextual conditioning are observed in different conditions (the contextual effect in the $10 \mathrm{sec}$ trace condition and the CS effect in the $30 \mathrm{sec}$ trace condition). Therefore, it might be maintained that these effects instead result from, first, an impairment of the overshadowing that normally takes place between environmental cues competing for association and, second, a delay-dependent deficit in the acquisition of conditioning to explicit cues (CSs). Thus, in the $10 \mathrm{sec}$ trace condition, contextual cues could be strongly conditioned in the lesioned animals despite strong conditioning of the CS in this group (abolished overshadowing). The CS conditioning deficit in the $30 \mathrm{sec}$ trace condition would then be interpreted as resulting from disruption of a separate mechanism.

There are several arguments against this alternative proposal. First, if only behavioral measures are considered (Fig. 1), ceiling effects can be invoked to explain the inability to observe both aspects of the attentional effect in one group of animals. Second, it has clearly been shown that the DNAB lesion-induced impairment in (CS) conditioned suppression can occur without a trace interval, when using a more sensitive lever-pressing paradigm involving multiple presentations of the CS (Tsaltas et al., 1983, 1984, 1989; Cole and Robbins, 1987), indicating that DNAB lesion-induced deficits in aversively motivated CS conditioning are not necessarily trace-dependent. Third, and most important, if neuroendocrine and behavioral data are considered together, enhanced contextual conditioning, measured in terms of behavior, and impaired CS conditioning, measured in terms of neuroendocrine response, are evident in the same (10 sec trace) condition.

The dual effects of DNAB lesions, impairment of CS conditioning and enhancement of contextual conditioning, could plausibly result from NA depletion in 2 separate terminal domains of the ceruleocortical noradrenergic projection. In fact, Winocur and collaborators (1987) have recently demonstrated a contextual conditioning effect, similar to that reported here, following dorsal hippocampal ablation. Other experimenters have also argued for the importance of hippocampal function in the processing of contextual cues (Nadel and Willner, 1980; Parkinson et al., 1988). On the other hand, a number of investigators have noted the importance of the amygdala for the maintenance of normal behavioral responses to conditioned aversive stimuli (Gallagher et al., 1977, 1981; McGaugh et al., 1984; Davis et al., 1987).

Whether the dual effects observed here depend on disruption of processing at more than one terminal locus of ceruleal noradrenergic projections cannot be determined at present. However, it is worth noting the conceptual and semantic problem posed by the distinction between CS and contextual cues for the electrophysiological analysis of locus ceruleus function described earlier. For example, because of the competitive relationship between CS and contextual cues, are the latter to be considered 
as "noise" within a mechanism processing the CS as a signal, or is the competition manifested by the interaction between outputs of distinct neural systems? The first of these possibilities may be especially exemplified by the electrophysiological data of Segal and Bloom (1976), who discovered that NA transmission can enhance the responsiveness of hippocampal cells to motivationally relevant signals (such as tone paired with reinforcement) in comparison with physically similar, but irrelevant, signals.

Regardless of the precise neuronal mechanism, it would appear in either case that the broadening of selective attention observed in the experiments reported here provides clear evidence that ceruleal noradrenergic projections normally function to enable the focusing of attention onto specific cues, predictive of reinforcers, in preference to the wider context in which they occur.

\section{References}

Aston-Jones, G. (1985) Behavioral functions of locus coeruleus derived from cellular attributes. Physiol. Psychol. 13: 118-126.

Breese, G. R., and T. D. Taylor (1971) Depletion of brain noradrenaline and dopamine by 6 -hydroxydopamine. Br. J. Pharmacol. 42: 88-99.

Carli, M., T. W. Robbins, J. L. Evenden, and B. J. Everitt (1983) Effects of lesions to ascending noradrenergic neurons on performance of a 5-choice serial reaction time task in rats: Implications for theories of dorsal noradrenergic bundle function based on selective attention and arousal. Behav. Brain Res. 9: 361-380.

Cole, B. J., and T. W. Robbins (1987) Dissociable effects of lesions to the dorsal or ventral noradrenergic bundle on the acquisition, performance and extinction of aversive conditioning. Behav. Neurosci. 101: $476-488$.

Crow, T. J. (1968) Cortical synapses and reinforcement: A hypothesis. Nature 219: 736-737.

Crow, T. J., J. F. W. Deakin, S. E. File, A. Longden, and S. Wendlandt (1978) The locus coeruleus noradrenergic system-Evidence against a role in attention, habituation, anxiety and motor activity. Brain Res. 155: 249-261.

Davis, M., J. M. Hitchcock, and J. B. Rosen (1987) Anxiety and the amygdala: Pharmacological and anatomical analysis of the fear-potentiated startle paradigm. In The Psychology of Learning and Motivation, G. Bower, ed., pp. 263-305, Academic, New York.

Everitt, B. J., T. W. Robbins, and N. R. W. Selden (1989) Functions of the locus coeruleus noradrenergic system: A neurobiological and behavioural synthesis. In The Pharmacology of Noradrenaline, C. A. Marsden and D. J. Heal, eds., Oxford U.P., Oxford (in press).

Foote, S. L., R. Friedman, and A. P. Oliver (1975) Effects of putative neurotransmitters on neuronal activity in monkey cerebral cortex. Brain Res. 86: 229-242.

Foote, S. L., F. E. Bloom, and G. Aston-Jones (1980) Impulse activity of coeruleus neurons in awake rats and squirrel monkeys is a function of sensory stimulation and arousal. Proc. Natl. Acad. Sci. USA 77: 3033-3037.

Foote, S. L., F. E. Bloom, and G. Aston-Jones (1983) Nucleus locus ceruleus: New evidence of anatomical and physiological specificity. Physiol. Rev. 63: 844-914.

Fuxe, K., T. Hökfelt, G. Jonsson, S. Levine, P. Lindbrink, and A. Lofstrom (1973) Brain and pituitary-adrenal interactions: Studies on central monoamine neurons. In Brain-Pituitary-Adrenal Interrelationships. V. Vertebrate Mechanisms for ACTII Secretion, pp. 239269, Karger, Basel.

Gallagher, M., B. S. Kapp, R. E. Musty, and P. A. Driscoll (1977) Memory formation: Evidence for a specific neurochemical system in the amygdala. Science 198: 423-425.

Gallagher, M., B. S. Kapp, J. P. Pascoe, and P. R. Rapp (1981) A neuropharmacology of amygdala systems which contribute to learning and memory. In The Amygdaloid Complex. INSERM Symposium, No. 20, Y. Ben-Ari, ed., pp. 343-354, Elsevier/North Holland, Amsterdam.

Gillies, G. E., and P. J. Lowry (1986) Adrenal function. In Neuroen- docrinology, S. L. Lightman and B. J. Everitt, eds., pp. 360-388, Blackwell Scientific, London.

Gray, J. A. (1982) The Neuropsychology of Anxiety, Clarendon, Oxford.

Grubbs, F. E. (1969) Procedures for detecting outlying observations in samples. Technometrics $11: 1-21$.

Hökfelt, T., O. Johansson, and M. Goldstein (1984) Central catecholamine neurons as revealed by immunocytochemistry with special reference to adrenaline neurons. In Handbook of Chemical Neuroanatomy, Vol. 2, Part 1, A. Bjorklund and T. Hökfelt, eds., pp. 157276, Elsevier, North Holland.

Jacobs, B. L. (1987) Brain monoaminergic unit activity in behaving animals. Prog. Psychobiol. Physiol. Psychol. 12: 171-206.

Kamin, L. J. (1969) Predictability, surprise, attention and conditioning. In Punishment and Aversive Behavior, B. A. Campbell and R. M. Church, eds., pp. 279-296, Appleton-Century-Crofts, New York.

Kasmatsu, T., and P. Heggelund (1982) Single cell responses in cat visual cortex to visual stimulation during iontophoresis of noradrenaline. Exp. Brain Res. 45: 317-324.

Kety, S. S. (1970) The biogenic amines in the central ncrvous system: Their possible roles in arousal, emotion and learning. In The Neurosciences: Second Study Program, F. O. Schmidt, ed., pp. 324-336, Rockefeller U. P., New York.

Kullback, S. (1968) Information Theory and Statistics, 2nd ed., Dover, New York.

Lightman, S. L., K. Todd, and B. J. Everitt (1983) Role for lateral tegmental noradrenergic neurons in the vasopressin response to hypertonic saline. Neurosci. Lett. 42: 55-59.

Lorden, J. F., E. J. Rickert, R. Dawson, and M. A. Pelleymounter (1980) Forebrain norepinephrine and the selective processing of information. Brain Res. 190: 569-573.

Mackintosh, N. J. (1975) A theory of attention: Variations in the associability of stimuli with reinforcement. Psychol. Rev. 82: 276298.

Marlin, N. A. (1980) Contextual associations in trace conditioning. Anim. Learn Behav. 9: 519-523.

Martin-Iverson, M. T., M. Pisa, E. Chan, and H. C. Fibiger (1982) Enhanced neophobia but normal plasma corticosterone in rats with dorsal noradrenergic bundle lesions. Pharmacol. Biochem. Behav. 17: 639-643.

Mason, S. T., and H. C. Fibiger (1978) Evidence for a role of brain noradrenaline in attention and stimulus sampling. Brain Res. 159 . $421-426$

Mason, S. T., and S. D. Iversen (1975) Learning in the absence of forebrain noradrenaline. Nature $258: 422-424$.

Mason, S. T., and S. D. Iversen (1979) Theories of the dorsal bundle extinction effect. Brain Res. Rev. 1: 107-137.

Mason, S. T., and D. Lin (1980) Dorsal noradrenergic bundlc and selective attention. J. Comp. Physiol. Psychol. 94: 819-832.

McGaugh, J. L., K. C. Liang, C. Bennett, and D. B. Sternberg (1984) Adrenergic influences on memory storage: Interaction of peripheral and central systems. In Neurobiology of Learning and Memory, G. Lynch, J. L. McGaugh, and N. M. Weinberger, eds., pp. 313-332, Guilford, New York.

Mefford, I. N. (1981) Application of high-performance liquid chromatography with electrochemical detection to neurochemical analysis: Measurement of catecholamines, serotonin and metabolites in rat brain. J. Neurosci. Methods 3: 207-224.

Meites, J., and W. E. Sonntag (1981) Hypothalamic hypophysiotrophic hormones and neurotransmitter regulation: Current views. Annu. Rev. Pharmacol. Toxicol. 21: 295-322.

Mezey, E., H. Z. Kiss, L. R. Skirboll, M. Goldstein, and J. Axelrod (1984) Increase of corticotrophin-releasing factor staining in rat paraventricular nucleus neurones by depletion of hypothalamic adrenaline. Nature 310: 140-141.

Nadel, L., and J. Willner (1980) Context and conditioning: A place for space. Physiol. Psychol. 8: 218-228.

Odling-Smee, F. J. (1975a) The role of background stimuli during Pavlovian conditioning. J. Exp. Psychol. 27: 201-209.

Odling-Smee, F. J. (1975b) Background stimuli and the interstimulus interval during Pavlovian conditioning. J. Exp. Psychol. 27:387-392.

Parkinson, J. K., E. A. Murray, and M. Mishkin (1988) A selective mnemonic role for the hippocampus in monkeys: Memory for the location of objects. J. Neurosci. 8: 4159-4167.

Pisa, M., and H. C. Fibiger (1983a) Intact selective attention in rats 
with lesions of the dorsal noradrenergic bundle. Behav. Neurosci. 97: 519-529.

Pisa, M., and H. C. Fibiger (1983b) Evidence against a role of the rat's dorsal noradrenergic bundle in selective attention and place memory. Brain Res. 272: 319-329.

Redmond, D. E. (1979) New and old evidence for the involvement of a brain norepinephrine system in anxiety. In Phenomenology and Treatment of Anxiety, W. G. Fann, I. Karacan, D. Pokorny, and R. L. Williams, eds., pp. 153-203, Spectrum, New York.

Rescorla, R. A., and A. R. Wagner (1972) A theory of Pavlovian conditioning: Variations in the effectiveness of reinforcement and non-reinforcement. In Classical Conditioning II: Current Research and Theory, A. H. Black and W. F. Prokasy eds., pp. 64-99, AppletonCentury-Crofts, New York.

Robbins, T. W., and B. J. Everitt (1982) Functional studies of the central catecholamines. Int. Rev. Neurobiol. 32: 303-365.

Robbins, T. W., B. J. Everitt, B. J. Cole, T. Archer, and A. Mohammed (1985) Functional hypotheses of the ceruleocortical noradrenergic projection: A review of recent experimentation and theory. Physiol. Psychol. 13: 127-1 50.

Roberts, D. C. S. (1981) An evaluation of the role of noradrenaline in learning. In Theory in Psychopharmacology, Vol. 1, S. J. Cooper, ed., pp 123-148, Academic, London.

Roberts, D. C. S., M. T. C. Price, and H. C. Fibiger (1976) The dorsal tegmental noradrenergic projection: An analysis of its role in maze learning. J. Comp. Physiol. Psychol. 90: 363-372.

Sawchenko, P. E., and L. W. Swanson (1982) The organization of noradrenergic pathways from the brainstem to the paraventricular and supraoptic nuclei in the rat. Brain Res. Rev. 4: 275-325.

Segal, M., and F. E. Bloom (1976) The action of norepinephrine in the rat hippocampus. IV. The effects of locus coeruleus stimulation on evoked hippocampal activity. Brain Res. 107: 513-525.

Tsaltas, E., G. C. Preston, and J. A. Gray (1983) The effects of dorsal bundle lesions on serial and trace conditioning. Behav. Brain Res. 10: 361-374.

Tsaltas, E., J. A. Gray, and M. Fillenz (1984) Alleviation of response suppression to conditioned aversive stimuli by lesions of the dorsal noradrenergic bundle. Behav. Brain Res. 13: 115-127.

Tsaltas, E., M. M. Schugens, and J. A. Gray (1989) Effects of lesions of the dorsal noradrenergic bundle on conditioned suppression to a CS and to a contextual background stimulus. Behav. Brain Res. 31 . 243-256.

Waterhouse, B. D., and D. J. Woodward (1980) Interaction of norepinephrine with cerebro-cortical activity evoked by stimulation of somatosensory afferent pathways. Exp. Neurol. 67: 11-34.

Winocur, G., J. N. P. Rawlins, and J. A. Gray (1987) The hippocampus and conditioning to contextual cues. Behav. Neurosci. 101:617-625. 